\title{
STATISTICAL METHOD FOR ASTROPHYSICAL TURBULENCE INVESTIGATION
}

\author{
A. LAZARIAN \\ DAMTP, University of Cambridge, UK
}

\begin{abstract}
Present-day galactic dynamo theories widely use a concept of the ISM turbulence. In the present paper a statistical method of MHD turbulence study is discussed. The method is based on the solution of the inverse problem for the observational data. It is shown that in many important cases the inverse problem has a unique analytical solution. Examples, including the study of a random density field and a random magnetic field, are discussed.
\end{abstract}

Key words: turbulence - statistical method - magnetic field

\section{The Method}

Astrophysical turbulence differs from that in a laboratory, partly because of extremely high the magnetic Reynolds numbers. The energy density of chaotic motions in the ISM is approximately equal to the energy of magnetic fields $\left(\sim 10^{-12} \frac{\mathrm{erg}}{\mathrm{cm}^{3}}\right)$. As physical processes in the ISM are extremely complex, none of the models known at present is able to describe this type of turbulence. The only possibility is to study the turbulence through observations, which provides a difficult problem. Although the turbulence results in fluctuations of different observable parameters, these fluctuations correspond only to the projected data. The problem of initial spectrum recovery is complicated by the fact that characteristics vary along the line of sight. Additional problems emerge due to the fact that at the point where the observer is sited and the information is collected, the projection is produced by converging lines of sight. Consequently, although the problem of astrophysical turbulence study through observations was formulated long ago (Kaplan et al. 1970) it was only recently that results have been obtained (Lazarian et al. 1987, Lazarian 1991, 1992a, b). At the moment, it is possible to state that there exists a new method of statistical study for the ISM turbulence. Unlike statistical procedures well-known in astrophysics (Kleiner et al. 1984), the method discussed here gives not just rough estimates of the characteristics involved but a detailed information about the properties of the ISM turbulence.

Density field. This field is the simplest one for investigation. For some important emission lines, e.g. the HI $21 \mathrm{~cm}$ line, intensity of the emission $I(1)$ is proportional to $\int_{0}^{L} n(x) \mathrm{d} x$, where $n(x)$ is a density emitting material. For such lines spectral characteristic $\mathcal{E}(k)=\int_{0}^{L} E(k, x) \mathrm{d} x$ (where $E(k, x)$ is a real 3D turbulence spectrum which vary along the line of sight $x$ ) can be expressed through a structure function of intensity $D(\theta)=\left\langle\left(I\left(\mathbf{l}_{1}\right)-I\left(\mathbf{l}_{2}\right)\right)^{2}\right\rangle$ (where $\cos \theta=\frac{\mathbf{l}_{1} \cdot \mathbf{l}_{2}}{\left|\mathbf{l}_{1}\right| \cdot\left|\mathbf{l}_{2}\right|}$ ) (Lazarian $1992 \mathrm{~b})$ as $\mathcal{E}(k)=æ \int_{0}^{\infty} k \theta J_{1}(k \theta R) \frac{\mathrm{d}}{\mathrm{d} \theta} D(\theta) \mathrm{d} \theta$, where $æ$ is a coefficient and $J_{1}(x)$ is a Bessel function of the first order. More complex relations have been derived (Lazarian, in preparation) when other (more complicated) relations between the accepted intensity and the density are valid. 
Magnetic field. For studying a random magnetic field, it is possible to use synchrotron intensity, Faraday rotation measures etc. All of these methods are useful as they give different combinations of random parameters. For example, synchrotron intensity is sensitive to a product of the squared perpendicular component of the magnetic field, $H_{\perp}^{2}$, over the density of cosmic rays $n_{r} .{ }^{1}$ Faraday measures can give $H_{\|} n_{\mathrm{e}}$, where $n_{\mathrm{e}}$ is the electronic density $H_{\|}$is parallel to the line of sight component of the magnetic field, etc. As statistical properties, for example, of $n_{e}$, can be found from independent observations, one approaches step by step to an adequate description of the ISM. For MHD turbulence study through fluctuations of the synchrotron radiation, the following relation is valid (Lazarian, in preparation):

$$
d_{\mathrm{e}}(r)-d_{t}(r)=æ_{1} \int_{\frac{r}{L}}^{\theta_{\max }} \frac{\theta \mathrm{d} \theta}{\sqrt{\theta^{2}-\frac{r^{2}}{L^{2}}}} \frac{\mathrm{d}}{\mathrm{d} \theta}\left(\frac{1}{\theta} \frac{\mathrm{d}}{\mathrm{d} \theta}\left(D_{2}(\theta) \cdot \theta\right)\right)+\mathcal{E}_{i}
$$

where $d_{e}(r)$ and $d_{t}(r)$ are longitudinal and transversal parts of the structure tensor of the random magnetic field, $æ_{1}$ is a coefficient, $D_{2}(\theta)$ is a quadrupole part of the structure function of synchrotron intensity, and $\mathcal{E}_{i}$ corresponds to small terms which can be taken into account in the iteration procedure. This result is obtained with the assumption that fluctuations of the cosmic ray density and the cosmic magnetic field are statistically independent. More complex relations are valid when these fields are interdependent. The appropriate solutions will be discussed elsewhere.

Discussion. A new method of the statistical study of the ISM turbulence is discussed. It is shown that it is possible to find statistical characteristics of different ISM random fields through observations. The information should contribute to our understanding of complex physical processes in the ISM, including the generation of magnetic fields. Although there are still many mathematical problems to solve (they include a proper mathematical description of the procedures of data analysis, problems related to different random field studies), the method is applicable even at this stage (Lazarian et al. 1991). It worth noting, that complexity of the derivation of the particular solution does not mean complexity in practical use of the method. For example, it has been shown recently (Lazarian 1992b) that spectral characteristics of random density field, $\mathcal{E}$, are related to a signal of interferometer, $S(u)$, in an extraordinary straightforward way: $\mathcal{E}(u) \sim S\left(\frac{u}{2 \pi}\right) u^{2}$, which makes the density field rather an easy object to study with the aid of an interferometer.

\section{References}

Kaplan, S.A. and Pickelner, S.B.: 1970, The Interstellar Medium, Harvard University Press.

Kleiner, S.C. and Dickman, R.L.: 1984, ApJ., 286, 255.

Lazarian, A. and Chibisov, G.V.: 1987, Preprint FIAN 283.

Lazarian, A. and Shutenkov, V.R.: 1991, Sov. Astron. Lett., 16, 297.

Lazarian, A.: 1991, in Fragmentation of Molecular Clouds and Star Formation, eds. E.Falgarone, F. Boulanger and G. Dubert, Kluwer, p. 65.

Lazarian, A.: 1992a, Astron and Astrophys Transactions, 3, 33.

Lazarian, A.: 1992b, in Proc. of the ESA Colloquium, Targets for Space-Based Interferometry, 13-16 October, Beaulieu, France.

${ }^{1}$ For simplicity, the cosmic rays index is adopted to be equal 3. 